\title{
Vasorelaxant Activities of Aqueous Extracts from Twenty Medicinal Plants Used in Oriental Medicines in Isolated Rat Aorta
}

\author{
Eun-Young Kim¹, Kyung-Ok Lee ${ }^{2}$, Dong-II $\mathrm{Kim}^{3}$, and Mee-Ra Rhyu ${ }^{1 \dagger}$ \\ ${ }^{1}$ Functional Food Technology Research Group, Korea Food Research Institute, Gyeonggi 463-746, Korea \\ ${ }^{2}$ Quality Control Lab, Hyundai Green Food Co., Ltd., Gyeonggi 448-503, Korea \\ ${ }^{3}$ Department of Obstetrics \& Gynecology, Dongguk University Ilsan Oriental Hospital, Gyeonggi 410-773, Korea
}

\begin{abstract}
Water extracts from 20 medicinal plants, traditionally used for postmenopausal symptoms in Korea, were examined for their vasorelaxant activity in isolated rat thoracic aorta rings precontracted with norepinephrine (NE). Among the 20 medicinal plants, Cornus officinalis $(\mathrm{CoEx}, 0.3 \mathrm{mg} / \mathrm{mL})$, Schisandra chinensis (ScEx, $0.3 \mathrm{mg} / \mathrm{mL})$, Erythrina variegate (EvEx, $0.3 \mathrm{mg} / \mathrm{mL})$, and Epimedium koreanum (EkEx, $0.3 \mathrm{mg} / \mathrm{mL}$ ) showed rapid relaxation of endothelium-intact aorta $(69 \pm 4 \%, 40 \pm 3 \%, 25 \pm 2 \%$, and $23 \pm 3 \%$ of active tone induced by $\mathrm{NE}$, respectively). In contrast, the extracts of Erythrina variegata (EvEx), Angelica gigas (AgEx), Pueraria thunbergiana (PtEx), and EkEx lead to gradual (i.e., long-term) relaxation to baseline in endothelium-intact vessels. The time to complete relaxation was $20 \sim 40 \mathrm{~min}$. These 6 plant extracts were selected for the investigation of possible underlying mechanisms. The CoEx-, ScEx-, or EkEx-induced rapid relaxations were virtually abolished by endothelium denudation, and were significantly inhibited by pretreatment with nitric oxide (NO) synthase inhibitor $\mathrm{N}^{G}$-nitro-L-arginine (L-NNA, $10 \mu \mathrm{M})$, indicating that increased formation of NO might contribute to the endothelium-mediated relaxation. In long-term responses, the endothelium denudation did not affect PtEx-induced relaxation, whereas it delayed responses by EvEx and AgEx, and significantly inhibited the effect of EkEx. Among EvEx, AgEx, and PtEx, EvEx attenuated the $\mathrm{CaCl}_{2}$-induced vasoconstriction in high-potassium depolarized medium, implying that EvEx is involved in inhibition of the extracellular calcium influx to smooth muscle through voltage dependent calcium channels. These results provide the scientific rationale for the interrelationships between the use of 20 medicinal plants and their effects on cardiovascular health in estrogen deficient conditions.
\end{abstract}

Key words: medicinal plants, rapid relaxation, long-term vasorelaxation

\section{INTRODUCTION}

Medicinal plants have been used as traditional remedies in Asian countries for hundreds of years. Current research efforts are narrowing the knowledge gap between the traditional and scientific use of plants in treating various health conditions. Asian clinical prescriptions are usually composed of a combination of medicinal plants to provide synergistic pharmacological effects for greater efficacy than a single plant used alone. The following 20 plants: Acanthopanax sessiliflorus, Aconitum carmichaeli, Angelica gigas, Astragalus membranaceus, Cnidium monnieri, Cornus officinalis, Cuscuta chinensis, Dioscorea japonica, Dipsacus asper, Epimedium koreanum, Erythrina variegata var. orientalis, Glycyrrhiza uralensis, Panax ginseng, Panax notoginseng, Polygonatum odoratum var. pluriflorum, Polygonatum stenophyllum, Polygonum multiflorum, Psoralea corylifolia, Pueraria thunbergiana, Schisandra chinensis, have an extensive history of medicinal use as ingredients in various prescriptions for managing menopause-related symptoms in Korea $(1,2)$. Despite their clinical use for alleviating menopausal symptoms, there is limited information available on their mechanism of action.

Phytoestrogens, a large family of plant-derived compounds, are similar in structure and function to estrogen (3). Phytoestrogens are believed to be important for the prevention of cardiovascular disease like estrogen (4), presumably due to their vasoprotective effect (5). In vitro experiments on vascular tissues have revealed that phytoestrogens produce endothelium-dependent or-independent relaxation, or both (6-8). Each of the 20 plants selected may provide phytoestrogens, which have a vasoprotective effect, based on its traditional uses in Korea. However, there has been no scientific data yet to show that they act on vascular tissues as phytoestrogens. The aim of this study was to examine the vasorelaxant effects in isolated aortic rings and possible mechanisms of action of 20 medicinal plants applied in various herbal pre- 
scriptions for managing menopause-related symptoms in Korean women.

\section{MATERIALS AND METHODS}

\section{Preparation of plant extracts}

All of the dried medicinal plants (Table 1) were purchased from a local market (Kyungdong Mart), and authenticated by Professor Yong Ki Park, Department of Oriental Medicine, College of Oriental Medicine, Dongguk University (Gyeongju, Korea). The 20 medicinal plants $(100 \mathrm{~g})$ were extracted consecutively under reflux with water $(1 \mathrm{~L})$ for $1 \mathrm{hr}$, evaporated under reduced pressure at low temperature $\left(37 \sim 40^{\circ} \mathrm{C}\right)$ and lyophilized. The solid was stored at $-20^{\circ} \mathrm{C}$ until use. A solution was prepared with distilled water at a concentration of $100 \sim 300$ $\mathrm{mg} / \mathrm{mL}$ on the day of the experiment. Each voucher specimen was deposited at the Korea Food Research Institute, Gyeonggi-Do, Korea (Table 1).

\section{Preparation of rat aortic rings}

Male Sprague-Dawley rats $(200 \sim 250$ g) were sacrificed by stunning and exsanguinations. The thoracic aorta was dissected free from the surrounding connective tissues and cut into rings $2 \sim 3 \mathrm{~mm}$ in length. The rings were then transferred to 4-mL horizontal-type muscle chambers, and bathed in physiological salt solution (PSS) containing (mM/L) $\mathrm{NaCl}$ (136.9), $\mathrm{KCl}$ (5.4), $\mathrm{CaCl}_{2}$ (1.5), $\mathrm{MgCl}_{2}$ (1.0), $\mathrm{NaHCO}_{3}$ (23.8), glucose (5.5), and EDTA $(0.01)$ at $37^{\circ} \mathrm{C}$, in an atmosphere of $95 \% \mathrm{O}_{2}$ and $5 \%$ $\mathrm{CO}_{2}$. The rings were mounted on stainless-steel hooks connected to a force-displacement transducer (FT 03; Grass, West Warwick, RI), connected to a polygraph system (RPS 212; Grass), and a computer analyzer (Power Laboratory 400, MacLab; AD Instruments, Castle Hill, Australia). A basal tension of $1 \mathrm{~g}$ was applied. Some segments were stripped of endothelium by gentle rubbing with a moistened swab. The functional state of the endothelium was verified by previous challenge of precontracted arteries with carbachol $(1 \mu \mathrm{M})(9)$. Each experiment was performed on rings prepared from different rats. All studies were performed according to the Guiding Principles for the Care and Use of Laboratory Animals of the Ethics Committee of the Korea Food Research Institute.

\section{Measurement of vascular reactivity of aortic rings}

All rings were equilibrated for 60 min under a resting tension of $1 \mathrm{~g}$ and then exposed repeatedly to $72 \mathrm{mM} / \mathrm{L}$ $\mathrm{KCl}$ PSS until the responses stabilized. A control contraction was then produced by addition of $300 \mathrm{nM}$ norepinephrine (NE). After sustained tension $(60 \%$ of the maximal contraction in response to $72 \mathrm{mM} \mathrm{KCl}$ PSS in endothelium-intact rings) was obtained, test solutions

Table 1. Botanical names, family, plant parts, voucher specimen number, and ethnomedical uses of twenty medicinal plants

\begin{tabular}{|c|c|c|c|c|}
\hline Botanical names & Family & Plant parts & $\begin{array}{c}\text { Voucher } \\
\text { specimen No. }\end{array}$ & Ethnomedical use \\
\hline $\begin{array}{l}\text { Acanthopanax sessiliflorus (RUPR } \\
\text { et MAX.) SEEM }\end{array}$ & Araliaceae & Cortex & AsEx-001 & $\begin{array}{l}\text { Antirheumatics or drugs for relieving } \\
\text { rheumatic conditions }\end{array}$ \\
\hline Aconitum carmichaeli DEBX & Ranunculaceae & Radix & AcEx-001 & Drugs for dispelling internal cold \\
\hline Angelica gigas NAKAI & Umbelliferaceae & Radix & AgEx-001 & Tonics for blood \\
\hline Astragalus membranaceus BUNGE & Leguminosae & Radix & AmEx-001 & Tonics for Qi \\
\hline Cnidium monnieri (L.) CUSSON & Umbelliferae & Fruit & CmEx-001 & Tonics for Yang-energy \\
\hline Cornus officinalis SIEB. et. ZUCC & Cornaceae & Fruit & CoEx-001 & Astringents \\
\hline Cuscuta chinensis LAM & Convolvulaceae & Semen & CcEx-001 & Tonics for Yang-energy \\
\hline Dioscorea japonica THUNB & Dioscoreaceae & Rhizoma & DjEx-001 & Tonics for Qi \\
\hline Dipsacus asper WALL & Dipsacaceae & Radix & DaEx-001 & Tonics for Yang-energy \\
\hline Epimedium koreanum NAKAI & Berberidaceae & Herb & EkEx-001 & Tonics for Yang-energy \\
\hline $\begin{array}{l}\text { Erythrina variegata var. } \\
\text { orientalis (L.) MERR }\end{array}$ & Leguminosae & Corex & EvEx-001 & $\begin{array}{l}\text { Drugs for relieving rheumatic } \\
\text { conditions and articular pain }\end{array}$ \\
\hline Glycyrrhiza uralensis FISCH & Leguminosae & Radix & GuEx-001 & Tonics for Qi \\
\hline Panax ginseng C.A.MEY & Araliaceae & Radix & PgEx-001 & Tonics for Qi \\
\hline Panax notoginseng BURK & Araliaceae & Radix & PnEx-001 & $\begin{array}{l}\text { Drugs for blood stagnation and } \\
\text { bleeding }\end{array}$ \\
\hline $\begin{array}{l}\text { Polygonatum odoratum var. } \\
\text { pluriflorum (Miq.) OHWI }\end{array}$ & Liliaceae & Rhizoma & PoEx-001 & Tonics for Yin-energy \\
\hline Polygonatum stenophyllum MAXIM & Liliaceae & Rhizoma & PsEx-001 & Tonics for Yin-energy \\
\hline Polygonum multiflorum THUNB & Polygonaceae & Radix & PmEx-001 & Tonics for blood \\
\hline Psoralea corylifolia LINNE & Leguminosae & Fruit & PcEx-001 & Tonics for Yang-energy \\
\hline $\begin{array}{l}\text { Pueraria thunbergiana (SIEB. et } \\
\text { ZUCC) BENTH }\end{array}$ & Fabaceae & Radix & PtEx-001 & Drugs for relieving spasmodic \\
\hline $\begin{array}{l}\text { Schisandra chinensis (TURCZ.) } \\
\text { BAILL }\end{array}$ & Schisandraceae & Fruit & ScEx-001 & Astringents \\
\hline
\end{tabular}


were added to the bath solution. The high-potassium solution was prepared by replacing the $\mathrm{NaCl}$ in PSS with an equimolar amount of $\mathrm{KCl}(10)$.

In experiments using nitric oxide synthase inhibitor, inhibitors were added $20 \mathrm{~min}$ before precontraction. The inhibitor of extract-induced endothelium-dependent relaxation tested was $\mathrm{N}^{G}$-nitro-L-arginine (L-NNA, $10 \mu \mathrm{M} /$ L). To examine the effects of the extracts on calcium influx, the endothelium-denuded arteries were washed three times at $10 \mathrm{~min}$ intervals with calcium-free medium containing EGTA $(1 \mathrm{mM})$. Then, the arteries were stimulated with calcium-free $72 \mathrm{mM} \mathrm{KCl}$ medium (with or without plant extracts) and increasing concentrations of $\mathrm{CaCl}_{2}(0.3 \sim 10 \mathrm{mM})$ were added. For comparison, the L-type calcium channel blocker nicardipine (5 and 10 $\mathrm{nM}$ ), instead of plants extracts, was evaluated in a separate series of experiments (11).

\section{Reagents}

Carbachol, L-NNA, nicardipine, and NE were purchased form Sigma Chemical Co. (St. Louis, MO, USA). Other chemicals used for the measurement of vascular reactivity were all reagent grades, and were dissolved in PSS

\section{Data analysis}

All results are expressed as means \pm SEM. The number of rings obtained from different rats is represented by $n$. Relaxation is expressed in terms of the percentage decrease in maximal contraction caused by NE (300 $\mathrm{nM}$ ). One-way ANOVA and the Student Newman Keul test were used for statistical analyses of differences be- tween groups and $\mathrm{p}$ values $<0.05$ were regarded as statistically significant.

\section{RESULTS AND DISCUSSION}

\section{Vasorelaxant effect of water extracts of 20 plants in endothelium-intact rat aortic rings}

The water extracts of 20 plants were assessed for effects on two phases relaxation in endothelium-intact rat aorta precontracted with NE $(300 \mathrm{nM})$. A rapid relaxation, which occurred within seconds following administration of the plant extracts, was seen in CoEx (69土 $4 \%), \operatorname{ScEx}(40 \pm 3 \%), \operatorname{EvEx}(25 \pm 2 \%)$, and EkEx (23 $\pm 3 \%)$ at $0.3 \mathrm{mg} / \mathrm{mL}$; the other extracts were under $20 \%$ (Table 2). CoEx, ScEx, and EkEx caused concentration dependent responses $\left(0.1 \sim 3.0 \mathrm{mg} / \mathrm{mL}\right.$; $\mathrm{IC}_{50}$ value: 0.18 , 0.79 , and $2.06 \mathrm{mg} / \mathrm{mL}$, respectively) (Fig. 1), while EvEx showed no concentration dependency $(0.3,1.0$, and 3.0 $\mathrm{mg} / \mathrm{mL}$ showed $25 \pm 2,42 \pm 3$, and $25 \pm 3 \%$ relaxation, respectively).

A second, slowly developing (i.e., long-term) relaxation to baseline, was seen in $\mathrm{AgEx}, \mathrm{EkEx}, \mathrm{EvEx}$, and PtEx. Among these, EvEx showed the shortest time to developing relaxation to baseline (18 $\mathrm{min})$, followed by $\mathrm{AgEx}$, PtEx, and EkEx (Fig. 2A). In general, long-term relaxation was not affected by the endothelium denudation, because it was mediated by smooth muscle direct mechanisms. However, the vasorelaxations evoked by natural products were mediated by multiple mechanisms: through endothelium-dependent, endothelium-independent, and membrane channels (12). The relaxation caused by

Table 2. Two phases relaxant effects of twenty medicinal plants

\begin{tabular}{|c|c|c|c|}
\hline \multirow{2}{*}{ Botanical names } & \multirow{2}{*}{$\begin{array}{c}\text { Water extracts } \\
\text { (Abbreviated name) }\end{array}$} & \multicolumn{2}{|c|}{ Vasorelaxant effect $(\%)$} \\
\hline & & Rapid & Long-term \\
\hline Acanthopanax sessiliflorus (RUPR et MAX.) SEEM & AsEx & $19 \pm 5$ & $26 \pm 13$ \\
\hline Aconitum carmichaeli DEBX & AcEx & $14 \pm 4$ & - \\
\hline Angelica gigas NAKAI & $\operatorname{AgEx}$ & $17 \pm 3$ & $100 \pm 1$ \\
\hline Astragalus membranaceus BUNGE & $\mathrm{AmEx}$ & - & - \\
\hline Cnidium monnieri (L.) CUSSON & $\mathrm{CmEx}$ & - & - \\
\hline Cornus officinalis SIEB. et. ZUCC & CoEx & $69 \pm 4$ & - \\
\hline Cuscuta chinensis LAM & $\mathrm{CcEx}$ & - & - \\
\hline Dioscorea japonica THUNB & $\mathrm{DjEx}$ & $10 \pm 5$ & - \\
\hline Dipsacus asper WALL & DaEx & - & - \\
\hline Epimedium koreanum NAKAI & EkEx & $23 \pm 3$ & $100 \pm 1$ \\
\hline Erythrina variegata var. orientalis (L.) MERR & EvEx & $25 \pm 2$ & $100 \pm 1$ \\
\hline Glycyrrhiza uralensis FISCH & GuEx & $17 \pm 3$ & $30 \pm 19$ \\
\hline Panax ginseng C.A.MEY & PgEx & - & - \\
\hline Panax notoginseng BURK & PnEx & - & $45 \pm 20$ \\
\hline Polygonatum odoratum var. pluriflorum (Miq.) OHWI & PoEx & - & - \\
\hline Polygonatum stenophyllum MAXIM & PsEx & - & - \\
\hline Polygonum multiflorum THUNB & PmEx & - & - \\
\hline Psoralea corylifolia LINNE & PcEx & $7 \pm 1$ & $50 \pm 10$ \\
\hline Pueraria thunbergiana (SIEB. et ZUCC) BENTH & PtEx & - & $100 \pm 1$ \\
\hline Schisandra chinensis (TURCZ.) BAILL & $\mathrm{ScEx}$ & $40 \pm 3$ & - \\
\hline
\end{tabular}


A.

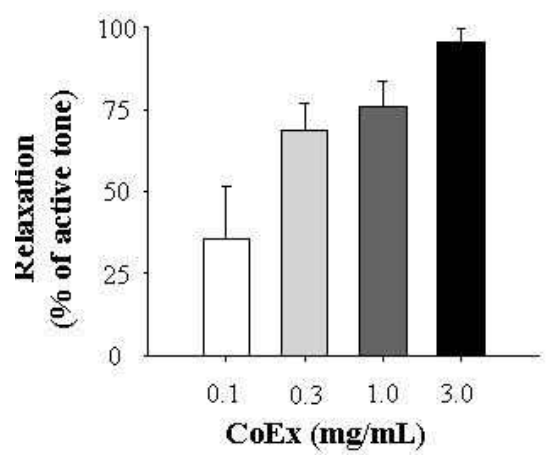

B.

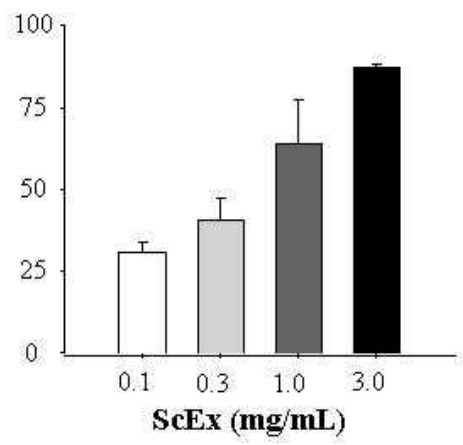

C.

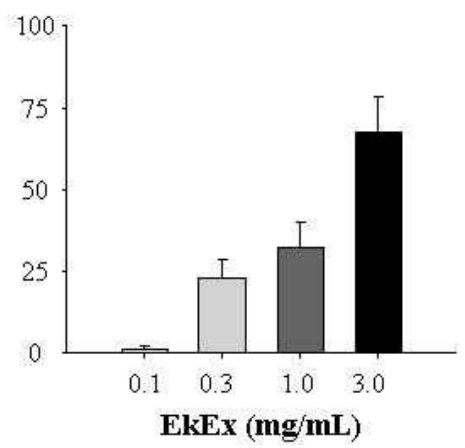

Fig. 1. The concentration dependency $(0.1 \sim 3.0 \mathrm{mg} / \mathrm{mL})$ of the extracts of Cornus officinalis (A, CoEx), Schisandra chinensis (B, ScEx), and Epimedium koreanum (C, EkEx). The ring contraction was induced with $300 \mathrm{nM}$ norepinephrine (NE) and the each extracts were added to the muscle. The relaxation responses are expressed as the percentage of NE-induced tone.

A.

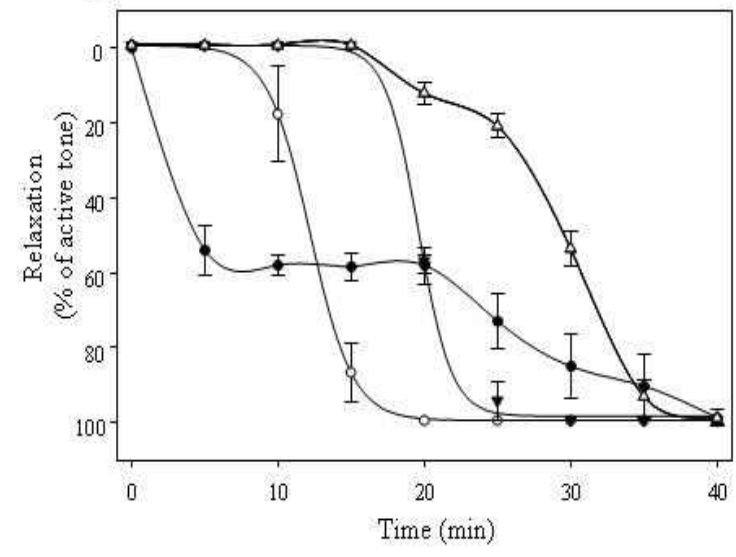

B.

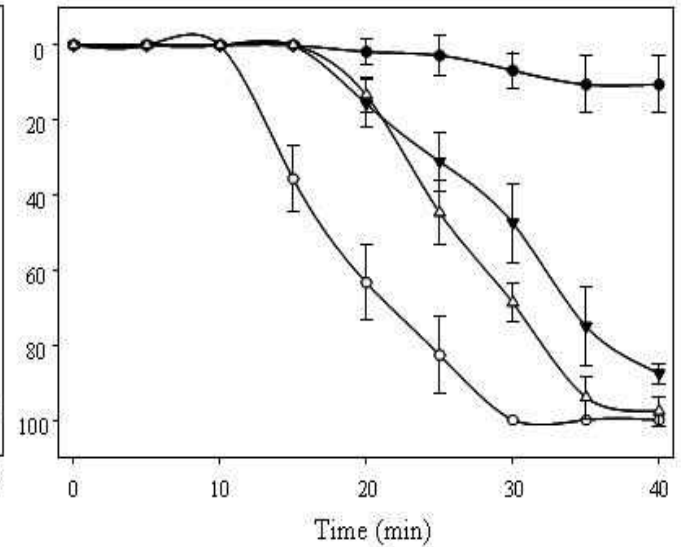

Fig. 2. The time-dependency of $\operatorname{AgEx}-(\boldsymbol{\nabla}, 0.3 \mathrm{mg} / \mathrm{mL})$, EkEx- $(\bullet, 0.3 \mathrm{mg} / \mathrm{mL})$, EvEx- $(\bigcirc, 0.3 \mathrm{mg} / \mathrm{mL})$, and PtEx- $(\triangle, 0.3$ $\mathrm{mg} / \mathrm{mL}$ ) induced vascular relaxation in the endothelium-intact (A) and -denuded (B) rat aorta. The relaxation responses are expressed as the percentage relaxation of the NE-induced contraction.

PtEx was not affected by endothelium denudation, whereas the response of EkEx was significantly inhibited (Fig. 2B). This result suggested that the effect of EkEx in long-term relaxation was not mediated by the smooth muscle direct pathway, but by endothelium related function. Additionally, AgEx- or EvEx-induced long-term relaxations were slightly delayed in endothelium-denuded rings, implying that these effects were elicited by both endothelium-dependent and -independent mechanisms. Therefore, to further elucidate the underlying mechanisms, we selected CoEx, ScEx, and EkEx for the representative extracts which elicit endothelium-dependent relaxation, and chose AgEx, EvEx and PtEx as endothelium-independent relaxant extracts.

\section{Mechanisms of selected medicinal plant extracts}

In rapid relaxation, the endothelium participates as an important regulator of vascular tone by releasing endothelium-derived relaxing factor (EDRF) (13). Many EDRFs are secreted from endothelium, the most potent molecule is probably nitric oxide (NO) (14-16). As shown in Fig. 3, CoEx-, ScEx-, or EkEx-induced vasorelaxations were abolished by endothelium denudation, and these rapid relaxations were significantly inhibited by pretreatment with L-NNA $(10 \mu \mathrm{M})$. Endothelial NO plays a major role in the control of vasomotor tone and structure (17). Under the pathological conditions of cardiovascular diseases, dysfunction exists in the vascular endothelium with a subsequent reduction in the release, bioavailability or action of NO. Thus, NO release and function is often decreased in cardiovascular diseases, such as hypertension (17), and atherosclerosis (18). We reported the vasorelaxant effect of Schizandra chinensis and Angelica gigas extracts $(19,20)$, and quite recently, showed the synergistic vasorelaxant effect of Ligusticum wallichii and Angelica gigas by stimulation of NO release (21). Sohn et al. (22) reported that medicinal plant extracts containing Cornus officinalis and Schisandra chinensis reduced blood pressure and increased NO syn- 
A.

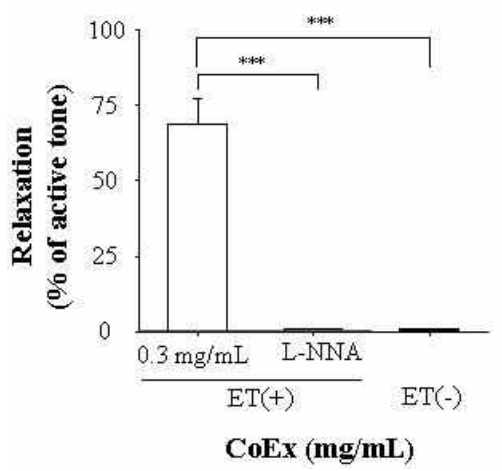

B.

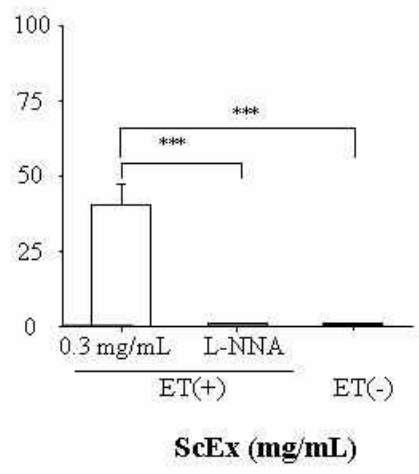

C.

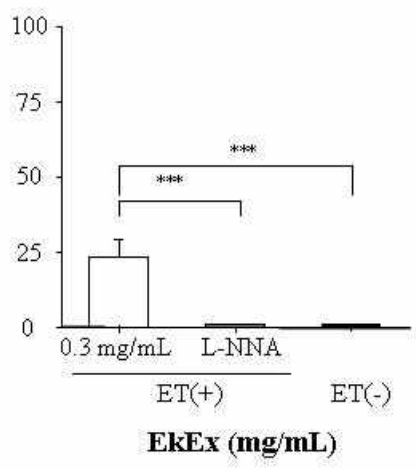

Fig. 3. Comparison of the responses to $\operatorname{CoEx}(\mathrm{A}), \operatorname{ScEx}(\mathrm{B})$, or EkEx (C) of endothelium-intact ( $\square$ ) and inhibitor treated rat

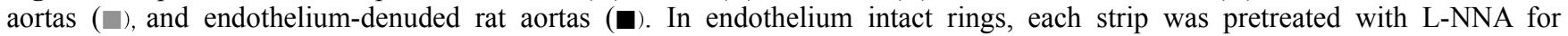
$20 \mathrm{~min}$ in the quiescent preparation, and each extract was added to NE-contracted aorta. The relaxation responses are expressed as the percentage of NE-induced tension.

thase in spontaneously hypertensive rats. Epimedium extract was reported to exert partial endothelial NO-dependent vasorelaxation in rat aorta (23). Additionally, many studies have attempted to identify plant extracts with vasorelaxant effects, and they found that the vasorelaxant effects are related to stimulation of NO release from vascular tissues (24-26). Therefore, CoEx, ScEx, or EkEx elicited the vasorelaxation via endothelial $\mathrm{NO}$ production, and these endothelium-dependent vasorelaxations may contribute to the beneficial effects on the cardiovascular system.

The influx and bioavailability of calcium is an important mediator of excitation-contraction coupling in smooth muscle cells, and inhibition of calcium influx is one of the major mechanisms in smooth muscle direct vasorelaxation (27). The rise of intracellular calcium concentration can be due to either $\mathrm{Ca}^{2+}$ entry through membrane channels, receptor-mediated $\mathrm{Ca}^{2+}$ release from the sarcoplasmic reticulum or both $(28,29)$. Transmembrane $\mathrm{Ca}^{2+}$ influx depends on the classical ionotropic function of ion channels, among which the L-type voltage-dependent calcium channels (VDCCs) are particularly relevant (30). We compared the effects of AgEx, EvEx, and PtEx on cumulative concentrations of $\mathrm{CaCl}_{2}$ $(0.3 \sim 10 \mathrm{mM})$ in a $72-\mathrm{mM} \mathrm{KCl} / \mathrm{L}$ depolarized calciumfree medium containing EGTA with the L-type calcium channel inhibitor, nicardipine. In our results, EvEx (0.3 $\mathrm{mg} / \mathrm{mL}$ ) significantly shifted the $\mathrm{CaCl}_{2}$-inducd contraction curves to the right and downward compared with a control to a similar level as nicardipine ( $5 \mathrm{nM} / \mathrm{L})$, but AgEx and PtEx did not (Fig. 4), suggesting that EvEx elicited the vasorelaxation via inhibition of calcium influx, especially through VDCCs. Although AgEx or PtEx did not affect the calcium-induced contraction in our experimental conditions, it is possible that these ex-

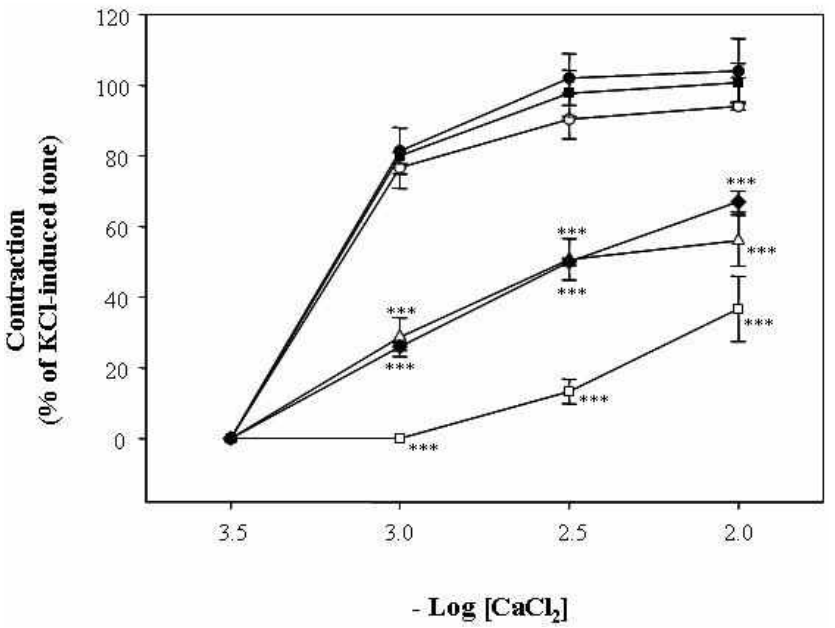

Fig. 4. The effects of $\operatorname{AgEx}(\bigcirc, 0.3 \mathrm{mg} / \mathrm{mL}), \operatorname{EvEx}(\triangle, 0.3$ $\mathrm{mg} / \mathrm{mL})$, and PtEx $(\boldsymbol{\square}, 0.3 \mathrm{mg} / \mathrm{mL})$ on calcium concentrationdependent contraction curves in rat thoracic aorta without endothelium compared to the effects of nicardipine $(\square, 5$ and $\downarrow, 10 \mathrm{nM} / \mathrm{L})$. Data are expressed as a percentage of the maximum contraction induced by $\mathrm{Ca}^{2+}$ in controls $(\bullet$, means \pm SEM). ${ }^{* * *} \mathrm{p}<0.001$ compared to the corresponding controls.

tracts produced the vasorelaxant effect by calcium related mechanisms other than extracellular calcium, such as intracellular release of calcium. The extract of Erythrina variegate exhibits beneficial effects against osteoporosis induced by estrogen deficiency through suppression of bone calcium turnover (31) and helps maintain calcium homeostasis (32). Therefore, the vasorelaxant effect mediated through calcium homeostasis, also, may be an important mechanism of the effect of EvEx in the management of menopausal symptoms. In these results, the inhibition of calcium influx was a possible mechanism of EvEx-induced vasorelaxation, but the other mechanisms of the EvEx-mediated effect may also contribute to the AgEx-, and/or PtEx-induced va- 
sorelaxation.

\section{CONCLUSION}

This study investigated the vasorelaxant effects and possible mechanisms of action of 20 medicinal plants, which have been used to manage postmenopausal symptoms in Korea. CoEx, ScEx, or EkEx elicited endothelial NO-dependent rapid vasorelaxation, and EvEx elicited long-term vasorelaxation via inhibition of calcium influx. Although the AgEx- and PtEx-induced vasorelaxation mechanisms were not identified in this study, it will be useful to further analyze those medicinal plants. These results may provide information on the cardio-protective effects of these herbs.

\section{ACKNOWLEDGEMENTS}

This study was supported by Technology Development Program for Agriculture and Forestry, Ministry for Agriculture, Forestry and Fisheries, Republic of Korea (GA0831) and the National Research Foundation of Korea Grant funded by the Ministry of Education, Science and Technology (KFR-2007-531-F00009).

\section{REFERENCES}

1. Donguibogam. 1999. Interlinear ed. Bubin Publishers Co., Seoul, Korea.

2. Kim DI, Woo DY, Seo JW, Lee TK. 2000. The management of climacteric syndrome clinic and the herbal medicine for that clinic. Kor J Orient Gynecol 13: 418-416.

3. Albertazzi P, Purdie DW. 2002. The nature and utility of the phytoestrogens: a review of the evidence. Maturitas 42: $173-185$.

4. Lissin LW, Cooke JP. 2000. Phytoestrogens and cardiovascular health. J Am Coll Cardiol 35: 1403-1410.

5. Ishimi $Y$, Miyaura $C$, Ohmura $M$, Onoe $Y$, Sato $T$, Uchiyama Y, Ito M, Wang X, Suda T, Ikegami S. 1999. Selective effects of genistein, a soybean isoflavone, on Blymphopoiesis and bone loss caused by estrogen deficiency. Endocrinol 140: 1893-1900.

6. Figtree GM, Griffiths H, Lu YQ, Webb CM, Macleod K, Collins P. 2000. Plant-derived estrogens relax coronary arteries in vitro by a calcium antagonistic mechanism. $J \mathrm{Am}$ Coll Cardiol 35: 1977-1985.

7. Mishra SK, Abbot SE, Choudhury Z, Cheng M, Khatab N, Maycock NJR, Zavery A, Aaronson PI. 2000. Endothelium-dependent relaxation of rat aorta and main pulmonary artery by the phytoestrogens genistein and daidzein. Cardiovasc Res 46: 539-546.

8. Lee MYK, Man RYK. 2003. The phytoestrogen genistein enhances endothelium-independent relaxation in the porcine coronary artery. Eur J Pharmacol 481: 227-232.

9. Sudjarwo SA, Hori M, Karaki H. 1992. Effect of endothelin-3 on cytosolic calcium level in vascular endothelium and on smooth muscle contraction. Eur J Pharmacol 229: 137-142.

10. Rhyu MR, Kim DK, Kim HY, Kim BK. 2000. Nitric ox- ide-mediated endothelium-dependent relaxation of rat thoracic aorta induced by aqueous extract of red rice fermented with Monascus rubber. J Ethnopharmacol 70: 29-34.

11. Salom JB, Burguete MC, Perez-Asenisio FJ, Centeno JM, Toregrosa G, Alborch E. 2002. Acute relaxant effects of $17-\beta$-estradiol through non-genomic mechanisms in rabbit carotid artery. Steroids 67: 339-346.

12. McNeill JR, Jrugens TM. 2006. A systematic review of mechanisms by which natural products of plant origin evoke vasodilatation. Can J Physiol Pharmacol 84: 803821.

13. Torok J. 2000. Histamine-induced relaxation in pulmonary artery of normotensive and hypertensive rats: relative contribution of prostanoids, nitric oxide and hyperpolarization. Pharmacol Res 49: 503-508.

14. Gryglewski RJ, Palmer RMJ, Moncada S. 1986. Superoxide anion is involved in the breakdown of endothelium-derived vascular relaxing factor. Nature 320: 454-456.

15. Lüscher TF. 1994. The endothelium in hypertension: bystander, target or mediator? J Hypertens 12: S105-S116.

16. Barton M, Cosentino F, Frandes RP, Moreau P, Shaw S, Lüscher TF. 1997. Anatomic heterogeneity of vascular aging: role of nitric oxide and endothelin. Hypertension 30: 817-824.

17. Lüscher TF, Vanhoutte PM. 1986. Endothelium-dependent contractions to acetylcholine in the aorta of the spontaneously hypertensive rat. Hypertension 8: 344-348.

18. Kanazawa K, Kawashima S, Mikami S, Miwa Y, Hirata K, Suematsu M, Hayashi Y, Itoh H, Yokoyama M. 1996. Endothelial constitutive nitric oxide synthase protein and mRNA increased in rabbit atherosclerotic aorta despite impaired endothelium-dependent vascular relaxation. $\mathrm{Am} J$ Pathol 148: 1949-1956.

19. Rhyu MR, Kim EY, Yoon BK, Lee YJ, Chen SN. 2006. Aqueous extract of Schizandra chinensis fruit causes endothelium-dependent and -independent relaxation of isolated rat thoracic aorta. Phytomedicine 13: 651-657.

20. Rhyu MR, Kim JH, Kim EY. 2005. Radix Angelica elicits both nitric oxide-dependent and calcium influx-mediated relaxation in rat aorta. $J$ Cardiovasc Pharmacol 46: 99104.

21. Kim EY, Rhyu MR. 2010. Synergistic vasorelaxant and antihypertensive effects of Ligusticum wallichii and Angelica gigas. J Ethnopharmacol 130: 545-551.

22. Sohn DW, Kim HY, Kim SD, Lee EJ, Kim HS, Kim JK, Hwang SY, Cho YH, Kim SW. 2008. Elevation of intracavernous pressure and NO-cGMP activity by a new herbal formula in penile tissues of spontaneous hypertensive male rats. $J$ Ethnopharmacol 120: 176-180.

23. Wang GJ, Tsai TH, Lin LC. 2007. Prenylflavonol, acylated flavonol glycosides and related compounds from Epimedium sagittatum. Phytochemistry 68: 2455-2464.

24. Kuramochi T, Chu J, Suga T. 1994. Gou-teng (from Uncaria rhynchophylla MIQUEL)-induced endotheliumdependent and -independent relaxations in the isolated rat aorta. Life Sci 54: 2061-2069.

25. Chen ZY, Zhang ZS, Kwan KY, Zhu M, Ho WKK, Huang Y. 1998. Endothelium-dependent relaxation induced by hawthorn extract in rat mesenteric artery. Life Sci 63: 1983-1991.

26. Chiou WF, Chang PC, Chou CJ, Chen CF. 2000. Protein constituents to the hypotensive and vasorelaxant activities of Cordyceps sinensis. Life Sci 66: 1369-1376. 
27. Meisheri KD, Hwang O, Breemen C. 1981. Evidence for two separate $\mathrm{Ca}^{2+}$ pathways in smooth muscle plasmalemma. $J$ Membrane Biol 59: 19-25.

28. Somlyo AP, Somlyo AV. 1994. Signal transduction and regulation in smooth muscle. Nature 372: 231-236.

29. Bolton TB, Prestwich SA, Zholos AV, Gordienko DV. 1999. Excitation-contraction coupling in gastrointestinal and other smooth muscles. Annu Rev Physiol 61: 85-115.

30. Urena J, Valle-Rodrigues A, Lopez-Barneo J. 2007. Metabotropic $\mathrm{Ca}^{2+}$ channel-induced calcium release in vascular smooth muscle. Cell Calcium 42: 513-520.

31. Zhang Y, Li XL, Lai WP, Chen B, Cow HK, Wu CHF, Wang NL, Yao XS, Wong MS. 2007. Anti-osteoporotic effect of Erythrina variegate L. in ovariectomized rats. $J$ Ethnopharmacol 109: 165-159.

32. Zhang Y, Li XL, Yao XS, Wong MS. 2008. Osteogenic activities of genistein derivatives were influenced by the presence of prenyl group at ring A. Arch Pharm Res 31: 1534-1539.

(Received April 27, 2010; Accepted September 10, 2010) 